\title{
A Prospective Observational Study of High-Flow Nasal Oxygen Therapy and Noninvasive Positive Pressure Ventilation in Patients with Acute Hypoxemic Respiratory Failure
}

\author{
Prashant Pandurang Jedge, Jignesh Navinchandra Shah, Shivakumar S. Iyer, Sampada Sameer Kulkarni \\ Department of Critical Care Medicine, Bharati Vidyapeeth (Deemed to be University) Medical College, Pune, Maharashtra, India
}

\section{Abstract}

Background: Treatment for acute hypoxemic respiratory failure (AHRF) includes treating the underlying disease, conventional oxygen therapy (COT), noninvasive ventilation (NIV), high-flow nasal oxygen (HFNO), and invasive mechanical ventilation. Aim: The aim of this study was to compare the use of HFNO and NIV in patients with moderate-to-severe AHRF to the tertiary level intensive care unit (ICU) of a teaching hospital. Methods: All adult patients admitted to the ICU with AHRF and failed COT were included. Administration of HFNO or NIV was protocol-based and targeted improvement in oxygen saturation, respiratory rate, $\mathrm{PaO}_{2}$, and $\mathrm{PaCO}_{2}$. Demographic data, clinical details, vital parameters, and laboratory findings were noted at prespecified intervals. Acute Physiology and Chronic Health Evaluation II at 24 h of ICU admission and daily Sequential Organ Failure Assessment were noted. The primary outcome was failure of treatment modalities defined as need for intubation and invasive ventilation. The secondary outcomes measured at 28 days were differences in ventilator-free days, ICU and hospital length of stay, patient comfort, and mortality. Results: A total of 35 patients were included in the study. Treatment failure was $20.8 \%(5 / 24)$ in the NIV group and $36 \%(4 / 11)$ in the HFNO group $(P=0.32)$. The number of ventilator-free days at day 28 was $22.67 \pm 9.92$ and $19.36 \pm 12.45(P=0.44)$ in the NIV and HFNO groups, respectively. Mortality at 28 days was $12.5 \%(3 / 24)$ and $27.2 \%(3 / 11)$ in the NIV and HFNO groups, respectively $(P=0.282)$. Conclusion: Treatment with HFNO is associated with nonsignificant increase in the need for intubation and 28-day mortality compared to NIV. Larger studies are required to assess the utility of HFNO in moderate-to-severe AHRF.

Keywords: Acute hypoxemic respiratory failure, high-flow nasal oxygen, noninvasive ventilation

\section{INTRODUCTION}

Treatment for acute hypoxemic respiratory failure (AHRF) includes treatment of underlying cause and supportive treatment in the form of conventional oxygen therapy (COT), noninvasive ventilation (NIV), high-flow nasal oxygen (HFNO), or invasive positive pressure ventilation. NIV was first used in the early 40s to treat patients with acute respiratory failure (ARF) ${ }^{[1]}$ Its use, however, has increased only in the last 30 years ${ }^{[2]}$ Currently, NIV refers to the delivery of positive pressure ventilation with techniques that do not need an invasive endotracheal airway. Class 1 indications for the use of NIV are reducing the need for intubation and mortality in chronic obstructive pulmonary disease $\mathrm{e}^{[3-5]}$ and AHRF due to acute cardiogenic pulmonary edema. ${ }^{[6]}$ The use of NIV for other causes of AHRF is still controversial, and there is no Class 1 evidence for the same. ${ }^{[7]}$ NIV is not suitable for patients who cannot protect their airway.

\begin{tabular}{|l|l|}
\hline \multicolumn{3}{|c|}{ Access this article online } \\
\hline Quick Response Code: & Website: \\
\hline & www.ijrc.in \\
\hline & \\
\hline
\end{tabular}

For patients with a weak cough reflex, or secretion, adequate suctioning and humidification is advisable before NIV is declared failed or contraindicated. In order to overcome some of the disadvantages of NIV, the novel technique of HFNO evolved in the last decade. As a result of humidification, HFNO improves mucociliary clearance ${ }^{[8]}$ HFNO reduces anatomic dead space by flushing out carbon dioxide from the proximal airways. ${ }^{[9]}$ Thus, it increases ventilatory efficiency and decreases the work of breathing. HFNO also provides

Address for correspondence: Dr. Jignesh Navinchandra Shah, F-1003, Venkatesh Lake Vista, Ambegaon, Pune - 411 046, Maharashtra, India.

E-mail: drshahjignesh@rediffmail.com

This is an open access journal, and articles are distributed under the terms of the Creative Commons Attribution-NonCommercial-ShareAlike 4.0 License, which allows others to remix, tweak, and build upon the work non-commercially, as long as appropriate credit is given and the new creations are licensed under the identical terms.

For reprints contact:WKHLRPMedknow_reprints@wolterskluwer.com

How to cite this article: Jedge PP, Shah JN, Iyer SS, Kulkarni SS. A prospective observational study of high-flow nasal oxygen therapy and noninvasive positive pressure ventilation in patients with acute hypoxemic respiratory failure. Indian J Respir Care 2021;10:201-5.

Received: $17-10-2020$ Accepted: 02-05-2021

Revised: 17-04-2021 Published: 14-06-2021 
minimal levels of positive end-expiratory pressure. ${ }^{[10]}$ Frat et al, in the 'FLORALI' trial in 2015, studied the efficacy of HFNO in patients of AHRF with a ratio of the partial pressure of arterial oxygen to the fraction of inspired oxygen $\left(\mathrm{PaO}_{2} / \mathrm{FiO}_{2}\right.$ ratio $)$ of $300 \mathrm{mmHg}$ or less. ${ }^{[11]}$

There are, however, limited data about the safety and efficacy of HFNO compared to NIV as first-line support in patients with moderate-to-severe AHRF not requiring immediate intubation.

The purpose of this study was to evaluate the use of NIV and HFNO in patients with moderate-to-severe AHRF of varied etiology.

\section{Methods}

A prospective observational study was conducted at a tertiary level intensive care unit (ICU) of a teaching hospital after institutional ethics committee approval. The study was conducted over a period of 15 months from September 2017 to November 2018. All patients with AHRF who failed the COT were included in the study. Failure of COT was defined by worsening tachypnea (respiratory rate $[R R]>35 / \mathrm{min}$ ), oxygen saturation $<88 \%$ on $\mathrm{FiO}_{2}$ of 0.6 on venturi mask, moderate/ severe hypoxemia defined as $\mathrm{PaO}_{2} / \mathrm{FiO}_{2}$ ratio $<200 \mathrm{mmHg}$, and signs of respiratory distress. Patients with type 2 respiratory failure $\left(\mathrm{PaCO}_{2}>45 \mathrm{mmHg}\right)$, chronic respiratory failure, chronic liver disease, and chronic kidney disease were excluded. Patients with contraindications to NIV such as hemodynamic instability (systolic blood pressure [BP] $<90 \mathrm{mmHg}$ or mean arterial pressure [MAP] $<65 \mathrm{mmHg}$ despite the use of vasopressors, i.e., noradrenaline $>0.1 \mathrm{mcg} / \mathrm{kg} / \mathrm{min}$ ), Glasgow Coma Score $(\mathrm{GCS})<12$, an urgent need for intubation, and life expectancy $<90$ days were also excluded. Informed valid written consent was taken. Patients were treated either NIV or HFNO depending on ICU physician's discretion and availability of the NIV or HFNO equipment. Administration of HFNO or NIV targeted improvement in saturation, RR, $\mathrm{PaO}_{2}, \mathrm{PaCO}_{2}$, and patient comfort. Settings of HFNO and NIV were adjusted as needed. Standard institutional protocol based on the FLORALI study for setting and monitoring of HFNO (Fisher and Paykel Healthcare - AIRVO-2) and NIV (NIV mode of conventional Ventilator - Vela) was used to guide the clinicians. ${ }^{[11]}$

In the HFNC group, AIRVO-2 (Fisher and Paykel Healthcare) was applied continuously through nasal prongs, with a flow rate of 40-60 L/min and initial $\mathrm{FiO}_{2}$ of $1.0 . \mathrm{FiO}_{2}$ was targeted to an oxygen saturation of $92 \%$ on HFNC. The patient was kept on HFNO for at least $2 \mathrm{~h}$. Once $\mathrm{FiO}_{2}$ requirement decreased below $50 \%$, the patient was switched to standard oxygen therapy based on clinical assessment. Intermittent removal of HFNO for nursing care was allowed as per standard care. These intervals were noted.

In the NIV group, NIV was applied through a face mask interface and used with an ICU ventilator (Vela). The patient was kept on NIV for at least $2 \mathrm{~h}$. Once deemed clinically stable with $\mathrm{FiO}_{2}$ requirements $<50 \%$, the patient was switched to standard oxygen therapy based on clinical assessment. Intermittent removal of NIV for nursing care was allowed as per standard care. The intervals were noted. At $2 \mathrm{~h}$ of therapy, patient comfort level was assessed with the Visual Analog Scale (VAS). It was assessed using an unmarked scale of 0-10 of VAS where 0 was the worst discomfort ever felt and 10 was no discomfort. The primary outcome was failure of treatment with HFNO or NIV. It was defined by need for intubation and invasive ventilation.

Standard criteria for endotracheal intubation and ventilation were used to guide the clinician to take decision for intubation. ${ }^{[11]}$

Worsening hemodynamic instability was defined as systolic BP $<90 \mathrm{mmHg}$ or MAP $<65 \mathrm{mmHg}$ despite the use of vasopressors (noradrenaline requirement $>0.1 \mathrm{mcg} / \mathrm{kg} / \mathrm{min}$ or dopamine requirement $>10 \mathrm{mcg} / \mathrm{kg} / \mathrm{min}$ ).

Worsening in neurological condition was identified by decrease in GCS by two points.

Worsening respiratory failure was identified as (any two):

a. Presence of excess secretions

b. Oxygen saturation $<88 \%$ as shown by pulse oximetry

c. Respiratory acidosis $(\mathrm{pH}<7.25)$

d. Respiratory distress identified as RR $>35 / \mathrm{min}$.

The secondary outcomes evaluated at 28 days were differences in ventilator-free days, ICU length of stay, hospital length of stay, and mortality.

For the purpose of this study, ventilated days were calculated from initiation of either NIV/HFNO to successful termination of either therapy. In patients who required invasive ventilation, the total number of ventilated days included the days of $\mathrm{NIV} / \mathrm{HFNO}$ and the days of invasive ventilation. Ventilator-free days at 28 days were calculated by subtracting total number of ventilated days from 28 . Patient comfort with either modality of intervention was observed using VAS. Probable causes of treatment failure were also evaluated. The incidence of complications such as septic shock, health-care-associated pneumonia (HCAP), and arrhythmias were observed.

Data were collected in a standardized pro forma for all patients included in the study. Demographic data such as patient identity, age, sex, and contact details were collected. Vital parameters and arterial blood gas (ABG) reports were recorded at specified intervals in the data collection sheet, i.e. at ICU admission, on failure of COT, $1 \mathrm{~h}$ and $24 \mathrm{~h}$ after the start of HFNO or NIV, and at the time of initiation of invasive mechanical ventilation in those patients who failed HFNO or NIV.

Clinical cause of hypoxemia was noted. Brain natriuretic peptide level, two-dimensional echocardiogram, and other tests were done if needed. Acute Physiology and Chronic Health Evaluation (APACHE) II was calculated at $24 \mathrm{~h}$ of admission 
Jedge, et al.: HFNO versus NIV for acute hypoxemic respiratory failure

for the severity of illness estimation. Sequential Organ Failure Assessment (SOFA) score was used in ICU on a daily basis for the $1^{\text {st }} 5$ days to assess the organ function in patients. For calculation of the neurological component of SOFA, the GCS score obtained before sedating the patient was used.

\section{Statistical analysis}

All the data were analyzed with IBM SPSS statistics software (IBM Corp. Released 2017. IBM SPSS Statistics for Windows, Version 25.0. Armonk, NY, USA: IBM Corp). Qualitative variables were compared with Chi-square test. Quantitative data variables between the groups were tested for the differences in the mean values with "unpaired $t$-test." Quantitative variables within the same groups (before and after intervention) were compared for the differences in means with "paired $t$-test." A two-tailed $P<0.05$ was considered to be statistically significant.

\section{RESULTS}

We studied 35 patients of AHRF of varied etiology who failed COT. Patients with predefined criteria were excluded from the study. Twenty-four patients received NIV and 11 patients received HFNO. One patient was crossover from HFNO to NIV on day 2 of the therapy [Figure 1]. All analysis was done on an intention-to-treat basis.

\section{Demographic and baseline characteristics}

The baseline characteristics of both the groups were comparable in terms of age, gender, baseline hemodynamic parameters, $\mathrm{PaO}_{2} / \mathrm{FiO}_{2}$ ratio, APACHE II at $24 \mathrm{~h}$, and mean duration of COT. The baseline characteristics are shown in Table 1. Figure 2 shows the etiology of AHRF in the HFNO and NIV groups.

The primary outcome was defined as need for intubation. Five out of 24 patients $(20.8 \%)$ failed NIV and required intubation while 4/11 (36.3\%) patients failed HFNO and were intubated. This difference in the primary outcomes was not statistically significant $(P=0.329)$ [Table 2].

\section{Secondary outcomes}

Three out of 24 patients died in the NIV group and 3/11 patients in the HFNO group $(P=0.282)$. Overall mortality was $17 \%$. Ventilator-free days at day 28 , average length of ICU stay and hospital stay, and patient comfort level were the other secondary outcomes studied in our study [Table 3]. Six patients in our study developed HCAP. Four out of 24 patients in the NIV group had HCAP (16.6\%) and 2/11 patients in the HFNO group had HCAP (18.1\%).

\section{Discussion}

There are limited randomised controlled trials comparing HFNO and NIV in AHRF in ICU settings. A recent systematic review by Lim Beng Leong compared HFNC versus NIV in adult ARF. ${ }^{[12]}$ The use of HFNO and NIV was in different settings than in our study and included periflexible bronchoscopy, postcardiac surgery, and patients who were at high risk of extubation failure. One study included in this meta-analysis by Stéphan et al. compared the use of HFNO and bilevel positive airway pressure (BiPAP) in ARF in patients who had undergone cardiothoracic surgery. They found that HFNO was not worse than NIV for the primary outcome of reintubation or premature discontinuation of therapy. ${ }^{[13]}$ Another study by Doshi et al., comparing NIV and high-velocity nasal insufflation for the treatment of respiratory failure in the emergency department, found that high-velocity nasal insufflation was noninferior to NIV for the treatment of respiratory failure in adult patients. ${ }^{[14]}$ The "FLORALI" trial, conducted in 23 ICUs across France and Belgium, included 310 patients and compared standard oxygen therapy with high-flow oxygen therapy and NIV. The study included AHRF patients with $\mathrm{PaO}_{2} / \mathrm{FiO}_{2}$ ratio of $<300 \mathrm{mmHg}$. In FLORALI study, the overall need for intubation was higher than in our study and was around 45\% (139/310). In the NIV group, the need for intubation was 50\% (55/110) which was higher than our study, and in the HFNO group, the need for intubation was $38 \%(40 / 106)$ which is lower than our study. The mean $\mathrm{PaO}_{2} / \mathrm{FiO}_{2}$ ratio in FLORALI study varied from

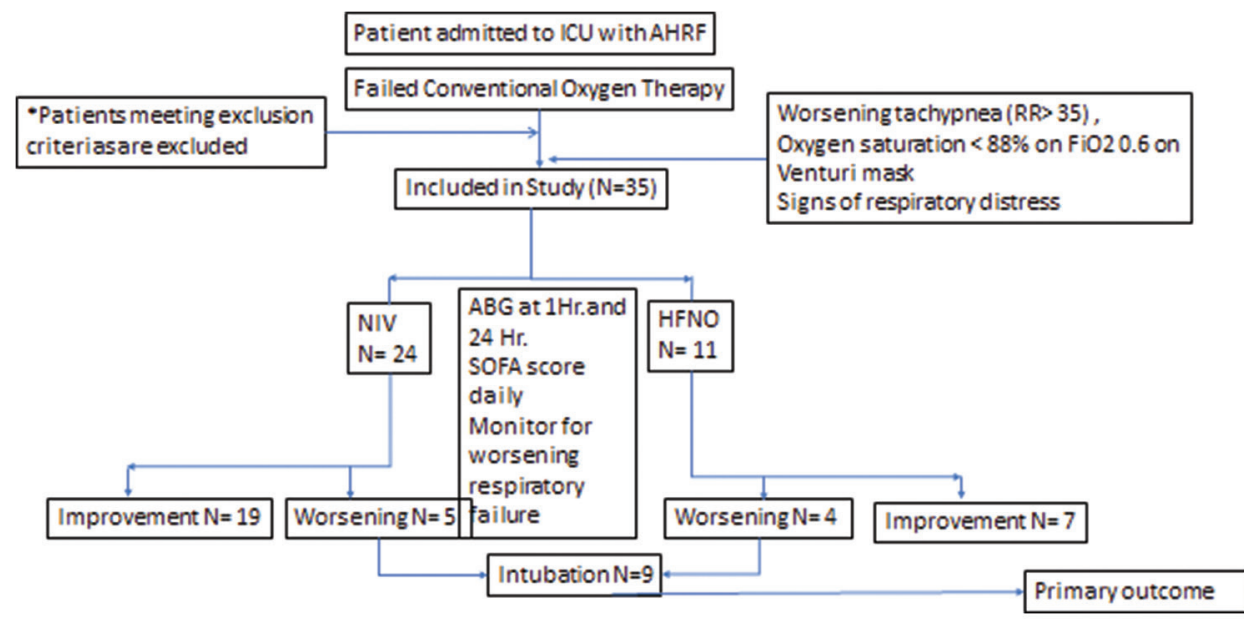

Figure 1: Consort diagram 
Jedge, et al.: HFNO versus NIV for acute hypoxemic respiratory failure

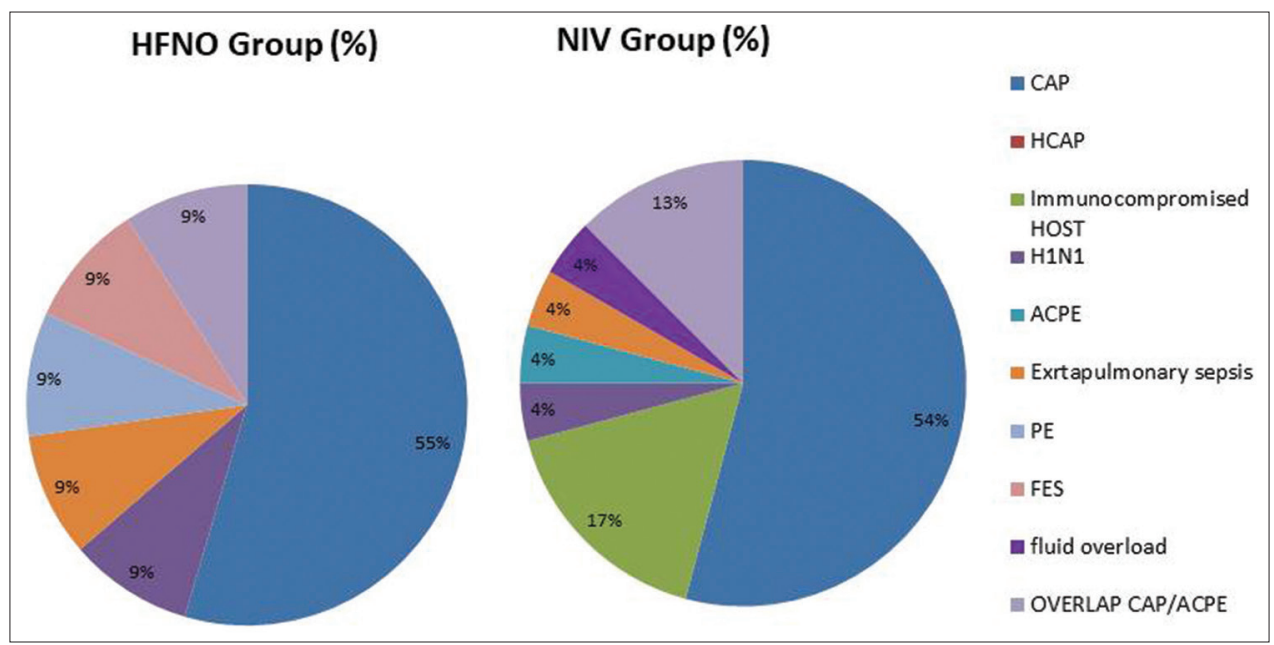

Figure 2: Etiology of acute hypoxemic respiratory failure

\section{Table 1: Baseline demographic and clinical characteristics of patients at inclusion}

\begin{tabular}{|c|c|c|c|}
\hline Baseline characteristics & NIV $(n=24)$ & HFNO $(n=11)$ & $P$ \\
\hline Male, $n(\%)$ & $16(66.6)$ & $7(63.6)$ & 0.86 \\
\hline Age (years) & $50.42 \pm 14.78$ & $50.42 \pm 16.85$ & 0.97 \\
\hline Heart rate (beats/min) & $106.42 \pm 16.80$ & $105.82 \pm 13.47$ & 0.91 \\
\hline Systolic blood pressure $(\mathrm{mmHg})$ & $126.96 \pm 34.48$ & $121.64 \pm 26.14$ & 0.62 \\
\hline Diastolic blood pressure (mmHg) & $78.29 \pm 16.81$ & $78.09 \pm 13.96$ & 0.97 \\
\hline $\mathrm{PaO}_{2} / \mathrm{FiO}_{2}$ at the entry in study $(\mathrm{mmHg})$ & $122.31 \pm 42.37$ & $108.6 \pm 37.03$ & 0.35 \\
\hline Lactates $(\mathrm{mg} / \mathrm{dL})$ & $2.42 \pm 2.06$ & $2.43 \pm 1.43$ & 0.99 \\
\hline Baseline RR (breaths/min) & $34.58 \pm 6.59$ & $33.55 \pm 7.03$ & 0.68 \\
\hline APACHE II at $24 \mathrm{~h}$ & $8.67 \pm 4.61$ & $8.0 \pm 3.41$ & 0.64 \\
\hline Mean duration of therapy (COT in hours) & $21.83 \pm 22.44$ & $18.73 \pm 19.79$ & 0.68 \\
\hline
\end{tabular}

$\mathrm{PaO}_{2}$ : Partial pressure of arterial oxygen, $\mathrm{FiO}_{2}$ : Fraction of inspired oxygen, COT: Conventional oxygen therapy, APACHE: Acute Physiology and Chronic Health Evaluation, NIV: Noninvasive ventilation, HFNO: High-flow nasal oxygen, RR: Respiratory rate

\begin{tabular}{lcc}
\hline \multicolumn{3}{l}{ Table 2: Primary outcome defined as need for intubation } \\
\hline Group & Need for intubation, $\boldsymbol{n}$ (\%) & $\boldsymbol{P}$ \\
\hline NIV & $5 / 24(20.8)$ & 0.329 \\
HFNO & $4 / 11(36.3)$ & \\
Total & $9 / 35(25.7)$ & \\
\hline
\end{tabular}

NIV: Noninvasive ventilation, HFNO: High-flow nasal oxygen

\section{Table 3: Secondary outcomes}

\begin{tabular}{lccc}
\hline Parameter & NIV & HFNO & $\boldsymbol{P}$ \\
\hline Mortality, $n$ (\%) & $3(24)$ & $3(11)$ & 0.282 \\
Ventilator-free days at day 28 & $22.67 \pm 9.09$ & $19.36 \pm 12.45$ & 0.44 \\
Length of ICU stay (days) & $7.04 \pm 6.58$ & $5.18 \pm 3.97$ & 0.31 \\
Length of hospital stay (days) & $14.33 \pm 10.07$ & $10.09 \pm 6.01$ & 0.13 \\
Patient comfort (VAS score) & $6.54 / 10$ & $7.09 / 10$ & 0.47 \\
\hline
\end{tabular}

NIV: Noninvasive ventilation, HFNO: High-flow nasal oxygen, ICU: Intensive care unit, VAS: Visual Analog Scale

149 to $161 \mathrm{mmHg}$. Our study looked at more severe AHRF with a mean $\mathrm{PaO}_{2} / \mathrm{FiO}_{2}$ ratio of $118 \mathrm{mmHg}$ at the time of inclusion in the study. Our study showed nonsignificant increase in the need for intubation for patients in the HFNC group as compared to the NIV group.
The present study was a prospective, observational study looking at patients with moderate-to-severe AHRF who failed COT and required either HFNO or NIV. There are no large-scale data comparing NIV and HFNO in this cohort of sick ICU patients, even though the use of HFNO is routine in patients with mild AHRF. Our study could look at all common etiologies of AHRF because we are a tertiary level ICU with wide case mix. Our study looked at the severity of illness of disease as a confounder for outcomes, and we monitored daily SOFA score for the first 5 days to look for progressive organ dysfunction.

However, the number of patients in our study was less as we excluded patients with acute exacerbation with chronic illnesses such as chronic liver disease, chronic respiratory disease, and chronic kidney disease. The study was observational and nonrandomized, and a number of patients in the NIV and HFNO groups were unequal due to resource constraints. The use of NIV or HFNO was left to the discretion and expertise of treating clinician and availability of device. NIV was administered with turbine-based invasive ventilator which has a dedicated NIV mode. It, however, had limited flow compensation, and we used passive humidifiers rather than heated humidification which is available on HFNO 
device. This may have been a confounder in the interpretation of our results.

\section{Conclusion}

In our prospective observational study of 35 patients with moderate-to-severe AHRF, HFNO was associated with nonsignificant increase in the need for intubation and 28-day mortality compared to the NIV group. Well-designed studies are required to assess the utility of HFNO in moderate-to-severe AHRF.

\section{Financial support and sponsorship}

Nil.

\section{Conflicts of interest}

There are no conflicts of interest.

\section{References}

1. Motley HL, Werko L. Observations on the clinical use of intermittent positive pressure. J Aviat Med 1947;18:417-35.

2. Pierson DJ. History and epidemiology of noninvasive ventilation in the acute-care setting. Respir Care 2009;54:40-52.

3. Brochard L, Mancebo J, Wysocki M, Lofaso F, Conti G, Rauss A, et al. Noninvasive ventilation for acute exacerbations of chronic obstructive pulmonary disease. N Engl J Med 1995;333:817-22.

4. Keenan SP, Sinuff T, Cook DJ, Hill NS. Which patients with acute exacerbation of chronic obstructive pulmonary disease benefit from noninvasive positive-pressure ventilation? A systematic review of the literature. Ann Intern Med 2003;138:861-70.

5. Lightowler JV, Wedzicha JA, Elliott MW, Ram FS. Non-invasive positive pressure ventilation to treat respiratory failure resulting from exacerbations of chronic obstructive pulmonary disease: Cochrane systematic review and meta-analysis. BMJ 2003;326:185.

6. Masip J, Roque $M$, Sánchez B, Fernández R, Subirana M, Expósito JA. Noninvasive ventilation in acute cardiogenic pulmonary edema: Systematic review and meta-analysis. JAMA 2005;294:3124-30.

7. Keenan SP, Sinuff T, Burns KE, Muscedere J, Kutsogiannis J, Mehta S, et al. Clinical practice guidelines for the use of noninvasive positive-pressure ventilation and noninvasive continuous positive airway pressure in the acute care setting. CMAJ 2011;183:E195-214.

8. Masclans JR, Pérez-Terán P, Roca O. The role of high flow oxygen therapy in acute respiratory failure. Med Intensiva 2015;39:505-15.

9. Dysart K, Miller TL, Wolfson MR, Shaffer TH. Research in high flow therapy: Mechanisms of action. Respir Med 2009;103:1400-5.

10. Parke R, McGuinness S, Eccleston M. Nasal high-flow therapy delivers low level positive airway pressure. Br J Anaesth 2009;103:886-90.

11. Frat JP, Thille AW, Mercat A, Girault C, Ragot S, Perbet S, et al. High-flow oxygen through nasal cannula in acute hypoxemic respiratory failure. N Engl J Med 2015;372:2185-96.

12. Beng Leong L, Wei Ming N, Wei Feng L. High flow nasal cannula oxygen versus noninvasive ventilation in adult acute respiratory failure: A systematic review of randomized-controlled trials. Eur J Emerg Med 2019;26:9-18.

13. Stéphan F, Barrucand B, Petit P, Rézaiguia-Delclaux S, Médard A, Delannoy $\mathrm{B}$, et al. High-flow nasal oxygen vs noninvasive positive airway pressure in hypoxemic patients after cardiothoracic surgery: A randomized clinical trial. JAMA 2015;313:2331-9.

14. Doshi P, Whittle JS, Bublewicz M, Kearney J, Ashe T, Graham R, et al. High-velocity nasal insufflation in the treatment of respiratory failure: A randomized clinical trial. Ann Emerg Med 2018;72:73-83. 